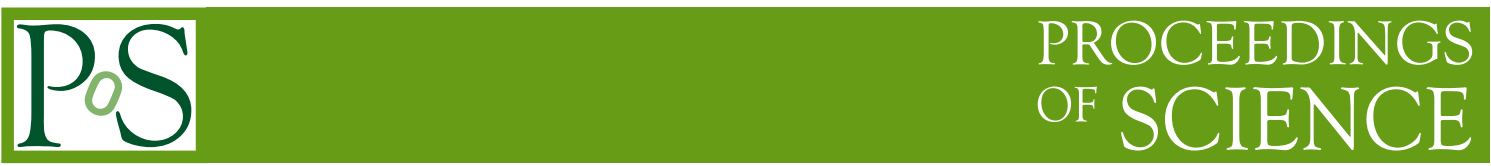

\title{
Measurement of Resonance Interaction in The NOMAD Detector
}

\author{
Hongyue Duyang* \\ University of South Carolina \\ E-mail: duyang@email.sc.edu
}

Xinchun Tian

University of South Carolina

E-mail: tainxcegmail.com

\section{Sanjib R. Mishra}

University of South Carolina

E-mail: mishra@barney.physics.sc.edu

This talk will be devoted to the precision measurements of charged current resonance (CC-Res) production. The new results from the NOMAD data will be presented, which is the most precise measurement so far. Future prospects of such measurements in the proposed high-resolution LBNE-ND will be outlined.

16th International Workshop on Neutrino Factories and Future Neutrino Beam Facilities 25 -30 August, 2014

University of Glasgow, United Kingdom

${ }^{*}$ Speaker. 


\section{Resonance Interactions and Motivation}

A neutrino can inelastically scatter off target nucleon via charged current interaction (CC), with a short term resonant state of the excited target nucleon created $\left(N^{*}, \Delta\right)$ and then almost immediately decays, most often into a nucleon and a single pion. The resonant states are predominately $\Delta^{++}$and $\Delta^{+}$:

$$
\begin{gathered}
v_{\mu}+p \longrightarrow \mu^{-}+\Delta^{++} \longrightarrow \mu^{-}+p+\pi^{+}, \\
v_{\mu}+n \longrightarrow \mu^{-}+\Delta^{+} \longrightarrow \mu^{-}+n+\pi^{+}, \\
v_{\mu}+n \longrightarrow \mu^{-}+\Delta^{+} \longrightarrow \mu^{-}+p+\pi^{0}
\end{gathered}
$$

Resonance interaction are described by Rein-Seghal (RS) model [1]. It assumes 3-quark system in relativistic harmonic oscillator potential, considering all resonance states with $W<2 \mathrm{GeV}$. A non-resonance, non-interfering background is added. The original RS model for RES assumes zero lepton mass. It shows some disagreement with the pion production data, especially in the low $Q^{2}$ region. The non-zero lepton mass effect was later introduced by Berger and Sehgal [2].

Resonance interaction is a large contribution to neutrino oscillation measurement in oscillation energy at few-GeV level, such as NOvA and LBNF at Fermilab, US. Although neutrino oscillation experiments use both a near and far detector to measure interactions before and after oscillation which reduces uncertainties from cross-sections, those uncertainties can not be completely canceled due to differences in detector efficiencies, flux and interactions between these two detector. The cross-section knowledge of resonance therefore is very important for those oscillation measurement.

\section{Introduction to NOMAD}

The Neutrino Oscillation MAgnetic Detector (NOMAD, WA-96) was designed to search for $v_{\mu}$ to $v_{\tau}$ oscillations in the CERN SPS wide band neutrino beam [3]. The neutrino beam was produced by the $450 \mathrm{GeV}$ protons from the Super Proton Synchrotron (SPS) incident on a beryllium target.The positively charged secondary $\pi, \mathrm{K}$ mesons were focused by two magnetic horns into a $290 \mathrm{~m}$ long evacuated decay pipe and then decayed into neutrinos.

The NOMAD detector was composed of several sub-detectors. The target consisted of 132 planes of $3 \times 3 \mathrm{~m}^{2}$ drift chamber (DC), with a 2.7 ton fiducial mass. The average density of $0.1 \mathrm{gm} / \mathrm{cm}^{3}$ is similar to that of liquid hydrogen, and the effective atomic number of 12.8 is similar to carbon. Following the drift chambers are a Transition Radiation Detector (TRD), a pre-shower detector (PRS) and a lead-glass electromagnetic calorimeter (ECAL). The ensemble of DC, TRD, and PRS/ECAL was placed within a dipole magnet providing a $0.4 \mathrm{~T}$ magnetic field orthogonal to the neutrino beam line, which makes possible high precision momentum measurement of charged particles.

During its run, the NOMAD experiment recorded over 1.7 million neutrino interactions in its active drift-chamber target. This high resolution neutrino data sample offers an unprecedented opportunity to study a large number of neutrino interactions in addition to the neutrino oscillation search. 


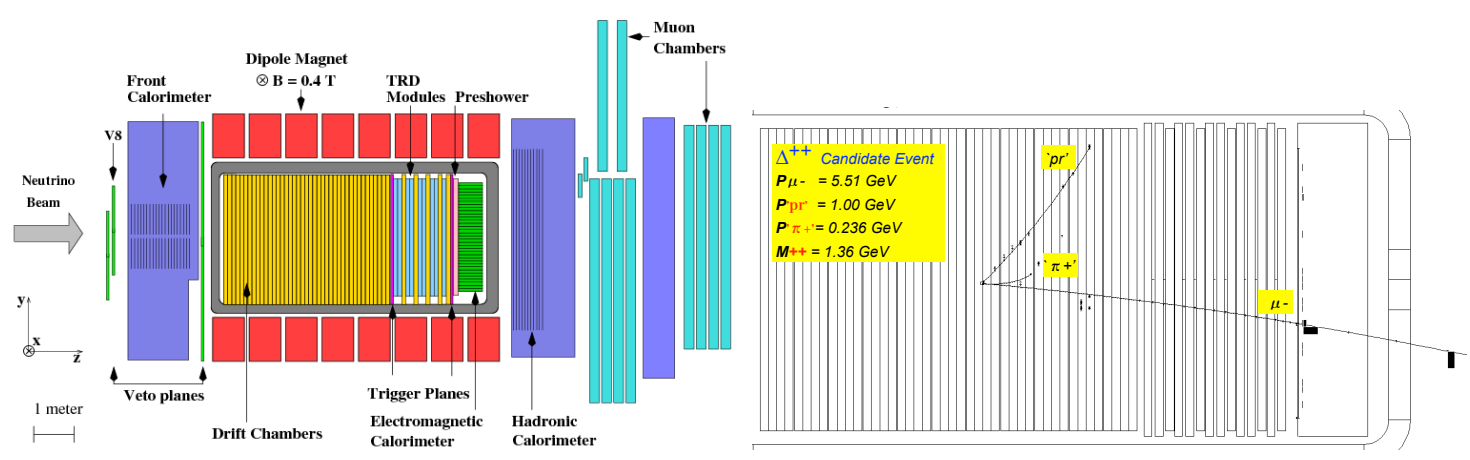

Figure 1: The NOMAD detector (left) and a candidate 3-track CC-Res event where a $\Delta^{++}$decays into a proton and $\pi^{+}$(right).

\section{Analysis}

We consider two independent samples of resonance interaction in NOMAD detector. The first is 3-track sample, in which a negative track from the muon, and two positive tracks from $\Delta^{++}$ decaying into a proton and $\pi^{+}$. The second sample is 2-track. It could be a negative muon track plus a positive track from decay of $\Delta^{++}$into proton or $\pi^{+}$, or one positive track missing from the 3-track sample.

The dominate background come from charged current deep inelastic scattering (CC-DIS). In a DIS event, a neutrino scatters off a quark in the nucleon via the exchange of a virtual $\mathrm{W}$ or $\mathrm{Z}$ boson, producing a lepton and a hadron shower in the final state. There can be 2 positive tracks of $\pi^{+}$or proton in the hadron shower besides the muon track, which mimic a 3 track RES signal. Other interactions, such as quasi-elastic $(\mathrm{QE})$ and coherent $(\mathrm{COH})$, is much smaller in number compared to DIS in 3-track sample, but may produce backgrounds to 2 track measurement.

Resonance interaction will first be measured using the two samples independently, and then combine to reduce statistics and systematic uncertainties and get a final result.

\subsection{Monte Carlo Simulation}

While NOMAD uses NEGLIB, built based upon LEPTO 6.1 and JETSET as its standard neutrino event generator, we used GENIE, a relatively modern neutrino generator to achieve better agreement with data. RS model is used in GENIE for resonance simulation. A GEANT program was used to simulate the NOMAD detector response [4]. All major interaction types are simulated.

For this analysis, the sample of MC events includes 50K RES. 1.35M CCDIS, 36K QE, 8K NCDIS and $7 \mathrm{~K}$ coherent. The total number of $\mathrm{CC}$ events is about $1.36 \mathrm{M}$ based on inclusive CC measurement [5] and the relative abundance of DIS:RES:QE was taken to be approximately 1.0:0.035:0.024.

\subsection{3-Track Sample Analysis}

- We select events in fiducial volume, requiring negative track identified as muon with 2 additional positive hadron track, and veto all photons to select the 3-track events. Pre-selection cuts are applied on kinematic variables such as missing $P_{T}$, total hadron energy $P_{\text {Had }}$ and hadron 
Table 1: Pre-selection cuts for 3-track analysis sample.

\begin{tabular}{|c|c|c|c|c|c|c|c|c|}
\hline Cuts & QE & RES & CCDIS & NCDIS & COH & TOT-Bac & MC & Data \\
\hline 3-Track in FV & 727.5 & 9836.4 & 70962.5 & 4.4 & 18.0 & 71712.4 & 81548.8 & 29568.0 \\
$P_{t}^{m}<0.7$ & 497.6 & 8326.4 & 36172.3 & 1.1 & 16.0 & 36687.0 & 45013.5 & 19800.0 \\
$\theta_{\text {had }}>0.35$ & 491.0 & 7467.1 & 16917.0 & 0.2 & 6.8 & 17415.0 & 24882.1 & 13982.0 \\
$p_{\text {had }}<2.5$ & 449.0 & 6999.2 & 12367.2 & 0.2 & 6.4 & 12822.8 & 19822.0 & 11992.0 \\
\hline
\end{tabular}
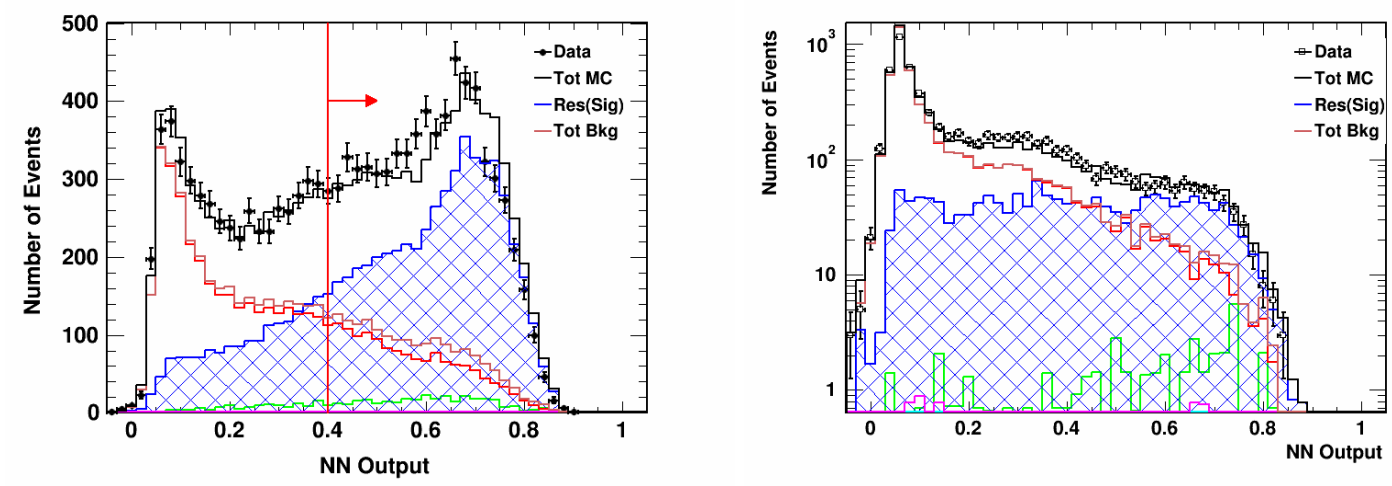

Figure 2: Neutral network output for 3-track analysis sample (left) and control sample (right) after background normalization.

angle from incoming neutrino $\theta_{\text {Had }}$ to further reduce backgrounds (table 1). The events selected are then subject to the neural network analysis.

The neural network has 9 input variables which are the 3-momentums (px, py, pz) of reconstructed muon, proton and pion tracks. The output is an number between 0 and 1 to separate signal from background. Signal region is defined as NN output greater than 0.4 where RES events dominates and background region smaller than 0.4 where the dominate is DIS. Background (DIS) is then normalized to fit data. Figure 2 compares NN output of data and MC, and figure 3 compares $Q^{2}$ and invariant mass of two hadron tracks. We find very good agreement. The fully-corrected signal events are measured to be 48,364 \pm 938 (statistics uncertainty).

A control sample is used to constrain DIS background in 3-track. One muon track, one positive hadron track and one negative hadron track. This sample is dominated by DIS. It is then subject to similar pre-selection cuts as in 3-track sample, and put into the same neural network. The data and MC comparison of the normalized NN output is shown in figure 2. The shape of the NN output well reproduces data in signal region (NNo $>0.4$ ). The DIS background is well under control.

\subsection{2-Track Sample Analysis}

A similar neutral network analysis is performed for 2-track events. Pre-selection cuts are applied to reduce background (table 2). The neural network takes 9 variables as input, which are $x_{b j}, y_{b j}, Q^{2}$ and the 3-momentums of muon and hadron track. Signal region is defined as NN output greater than 0.65 and background region smaller than 0.65 . The fully-corrected signal events are $47,386 \pm 1075$ (statistics uncertainty). 

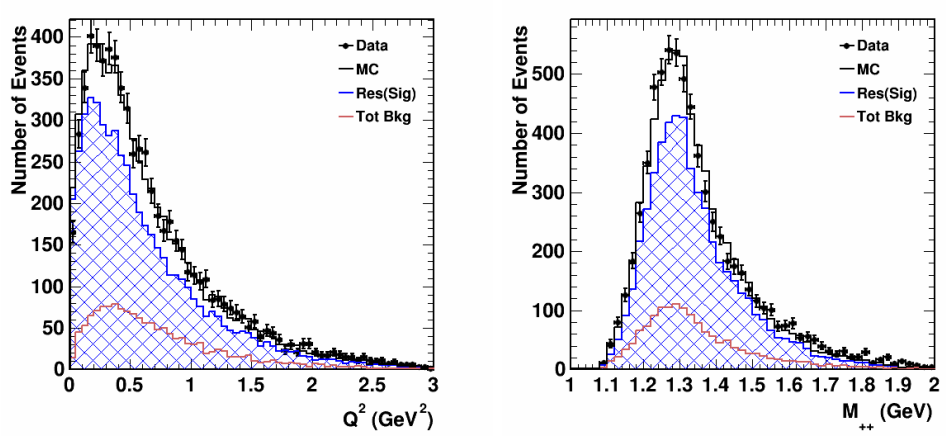

Figure 3: $Q^{2}$ and invariant mass comparison between 3-track data and MC sample in signal region after background normalization.

Table 2: Pre-selection of 2-track analysis sample.

\begin{tabular}{|c|c|c|c|c|c|c|c|c|}
\hline Cuts & QE & RES & CCDIS & NCDIS & COH & TOT-Bac & MC & Data \\
\hline 2-Track in FV & 11736.2 & 18212.3 & 73774.8 & 3.1 & 4044.8 & 89558.9 & 107771.3 & 46391.0 \\
$0.35<\theta_{\text {Had }}^{\mu}<1$ & 4000.5 & 12767.7 & 47978.3 & 0.9 & 1609.2 & 53588.9 & 66356.5 & 27707.0 \\
$Q^{2}<1 \mathrm{GeV}$ & 2042.5 & 9730.9 & 17084.5 & 0.7 & 1572.0 & 20699.8 & 30430.7 & 15817.0 \\
\hline
\end{tabular}

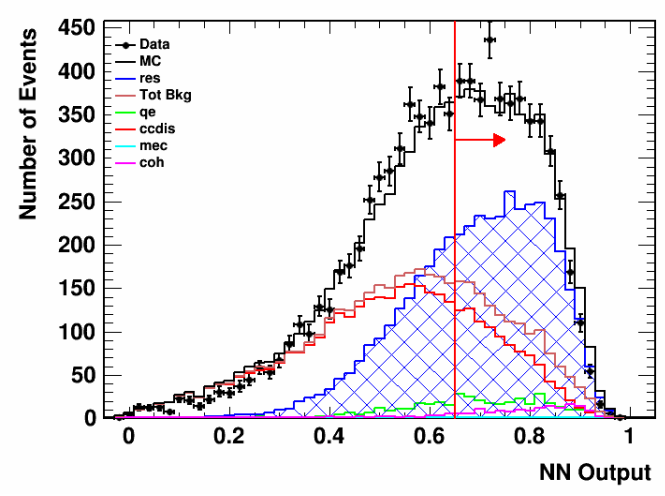

Figure 4: NN 2-track

\subsection{Combined Analysis and Systematic Uncertainties}

Adding up 3-track and 2-track analysis we get a combined result of inclusive RES measurement. The combined analysis not only increases statistics, but also less prone to systematic uncertainty. The final fully-corrected signal RES events are 47,942 \pm 207 . Figure 5 shows the ratio of RES with respect to inclusive $v_{\mu}$-CC and the RES cross-section as function of neutrino energy. The inclusive $v_{\mu}$-CC and NOMAD flux are obtained from previous NOMAD measurements [5].

Systematic uncertainties of this analysis comes from signal events selection, physics modeling of RES in MC simulations, as well as the flux measurement. The selection and simulation uncertainties can be evaluated by varying the cuts and physics constant values by one sigma, and 

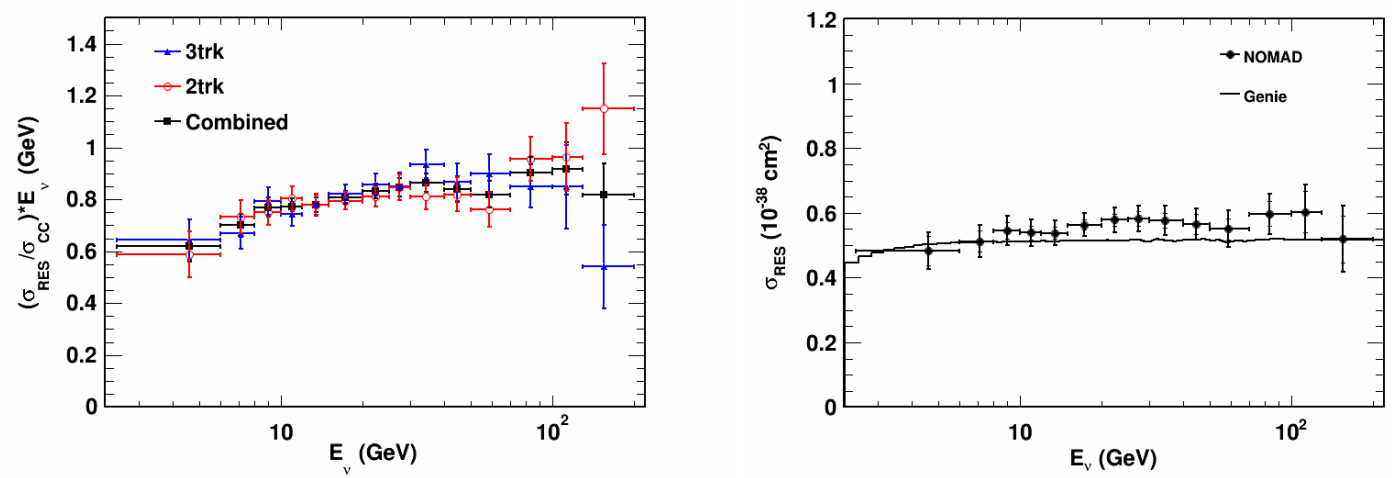

Figure 5: Ratio of measured resonance interaction to inclusic charged current $v_{\mu}$ interactions (left) and cross-section of meausured RES compared to GENIE prediction.

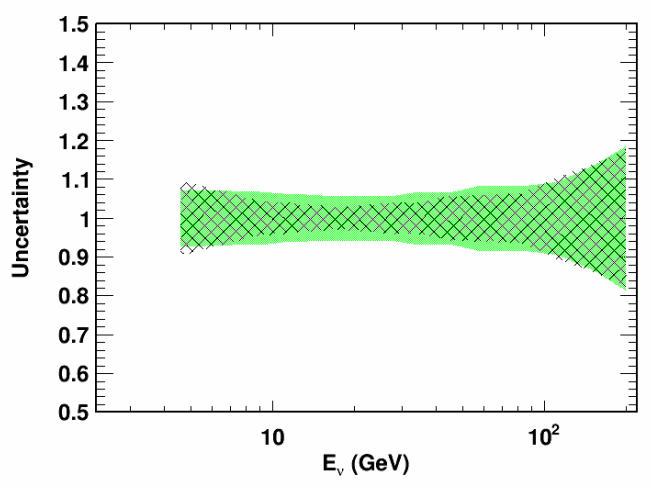

Figure 6: Statistic (hatch) and systematic (green) uncertainties in percentage.

calculate the variation in the final result. In this way they are determined to be $1.3 \%$ and $5.0 \%$ respectively. Flux uncertainty is obtained from previous measurement to be $4.1 \%$ [5]. Combining the uncertainty sources give a total systematic uncertainty of $6.6 \%$.

\subsection{Backward-Going Pions}

In the 3-track sample analysis, backward-going $\left(p_{z}<0\right)$ pions are of special interest. Pion momentum are most sensitive to nuclear effects. By looking at backward-going pions measured by data comparing with predicted by $\mathrm{MC}$, we get a handle on the nuclear effects simulation in neutrino event generator. Figure 7 shows such comparison in signal region of 3-track analysis. While data and MC agree well for forward-going pions, the agreement for backward-going pions is much worse. This shows that the backward-going pions are not well described by Genie and better understanding of nuclear effect in simulation is needed. 

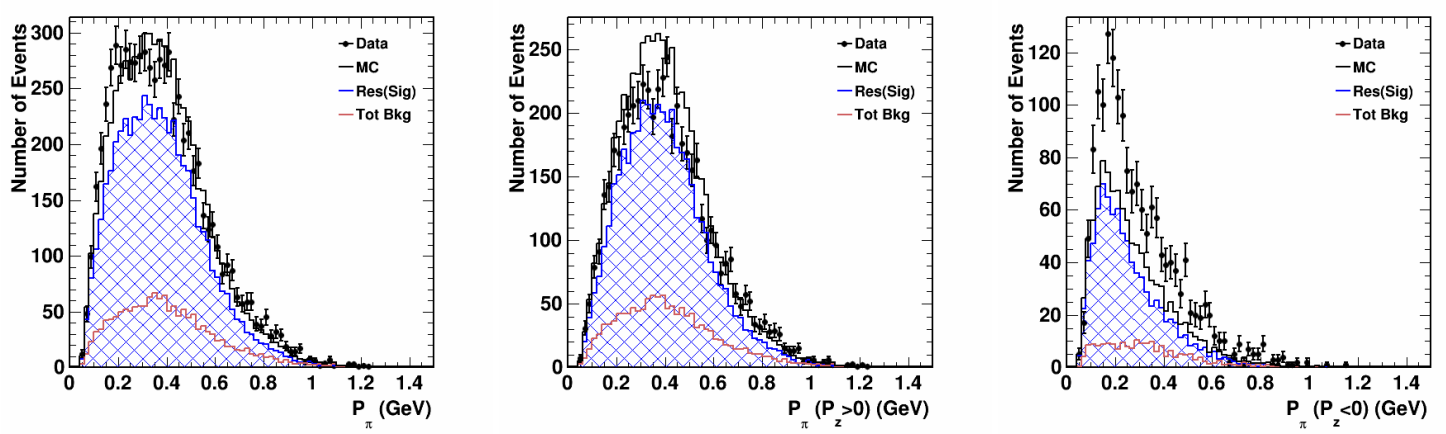

Figure 7: Momentum data and MC comparison of all pions (left), forward-going pions (middle), and backward-going pion (right).
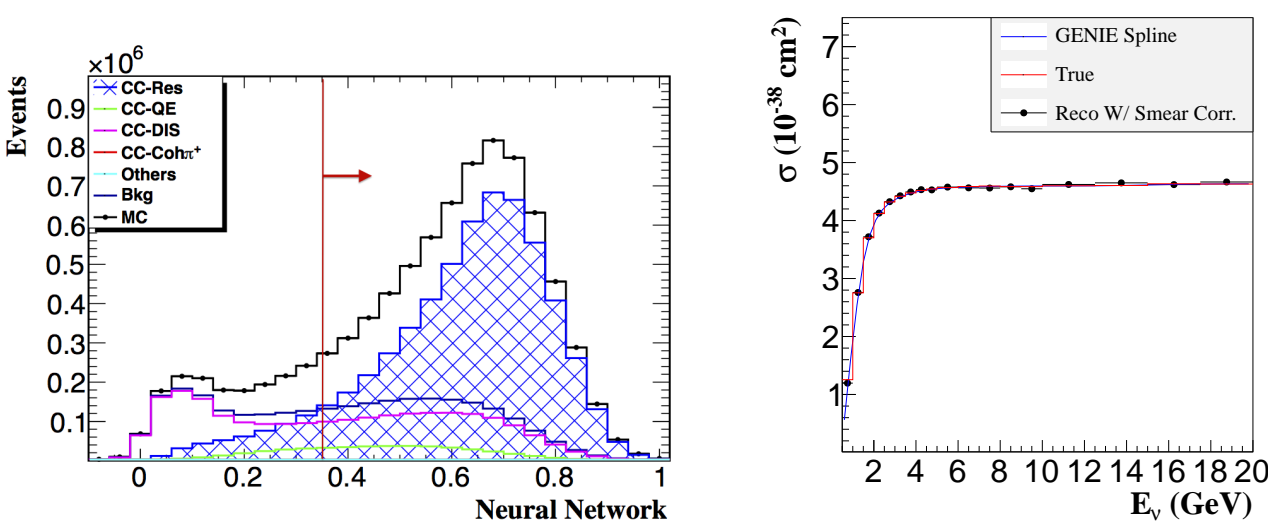

Figure 8: Resonance study in the proposed FGT detector for LBNF. The left figure shows the neural network performance, and the right one shows the measured cross-section using MC.

\section{Resonance In The Proposed FGT Detector for LBNF}

A FGT detector has been proposed as the near detector for Long-Baseline Neutrino Facility (LBNF) at Fermilab based on similar technology as the NOMAD detector. An similar multivariate neural network analysis for resonance interaction can be performed on the LBNF FGT detector to learn background characteristics, constrain the energy-scale of the neutrino, and constrain nuclear effects, and the NOMAD study serve as an important validation of the detector performance. A preliminary study using MC shows the neural network performance and the measured resonance cross-section can be found in figure 8 .

\section{Conclusion}

We have conducted a measurement of charged current resonance production. The analysis is data-driven. Two topologies, 3-track and 2-track, are included in the analysis. The backgrounds are constrained using a control sample. A total number of 47,942 \pm 719 (stat) \pm 3164 (syst) fullycorrected RES events are measured in NOMAD detector, which leads to the inclusive cross-section 
of RES being $0.559 \times 10^{-38} \mathrm{~cm}^{3}$. The ratio of RES to inclusive CC $\left(\sigma_{R E S} / \sigma_{C C} \times E_{V}\right)$ is measured to be $0.832(\mathrm{GeV})$. This is the most precise measurement of resonance interaction so far. It also serves as an important benchmark to validate future neutrino detector designs such as the proposed FGT detector for LBNF.

\section{References}

[1] D. Rein and L. M. Sehgal, Annals Phys. 133, 79 (1981).

[2] C. .Berger and L. M. Sehgal, Phys. Rev. D 76, 113004 (2007) [arXiv:0709.4378 [hep-ph]].

[3] J. Altegoer et al. [NOMAD Collaboration], NIM A404, 96-128 (1998)

[4] R.Brun et al., CERN Program Library, W5013, 1993.

[5] Q. Wu et al. [NOMAD Collaboration], Phys. Lett. B 660, 19 (2008) [arXiv:0711.1183 [hep-ex]]. 